\title{
OCCURRENCE OF EARLY ADVERSE EVENTS AFTER VACCINATION AGAINST INFLUENZA AT A BRAZILIAN REFERENCE CENTER
}

\author{
Marta Heloísa Lopes, Melissa Mascheretti, Marilia Miranda Franco, Ricardo \\ Vasconcelos, Eliana Battaggia Gutierrez
}

\begin{abstract}
Lopes MH. Mascheretti M. Franco MM. Vasconcelos R. Gutierrez EB. Occurrence of early adverse events after vaccination against influenza at a Brazilian reference center. Clinics. 2008;63(1):21-6.
\end{abstract}

INTRODUCTION: Since 1999, the Ministry of Health in Brazil has conducted campaigns of vaccination against influenza targeted towards the elderly, chronically-diseased people and health care workers. The vaccine against influenza is associated with adverse events of minor importance.

OBJECTIVE: To investigate the early adverse events related to the vaccine against influenza.

CASUISTICS AND METHODS: One hundred and ninety seven elderly individuals and health care workers vaccinated against influenza were included. An inquiry regarding adverse events related to the vaccine was applied seven days after the vaccination. RESULTS: Local adverse events were reported by $32.5 \%$ and systemic effects by $26.4 \%$ of the vaccinated subjects. Pain in the region of the injection, headache, myalgia, malaise, and coryza were more frequent in the workers than in the elderly $(\mathrm{p}<0.05$ ). There was no statistically significant difference in the occurrence of fever.

CONCLUSIONS: The belief of part of the population that credits frequent and uncomfortable adverse events to the vaccine was not confirmed. The subjective adverse events were more frequent in the health care workers, which can influence, in a negative way, the disclosure of the benefits of this vaccine due to their role as opinion makers.

KEYWORDS: Influenza vaccine. Adverse events. Elderly. Health care workers.

\section{INTRODUCTION}

Influenza is an acute respiratory disease that is autolimited and caused by Orthomyxovirus, predominantly the $\mathrm{A}, \mathrm{B}$ and $\mathrm{C}$ types. It is highly contagious, and infects a great number of individuals in a short period of time. In regions of cold and mild weather, the infections are more frequent during the winter months. Although some authors believe there is evidence of influenza epidemics dating back to $1510,{ }^{1}$ studies with serologic evidence became possible due to the isolation of the virus in $1933 .^{2}$

The Influenza viruses, mainly the A type, acquire fre-

Hospital das Clínicas, Faculdade de Medicina da Universidade de São Paulo - São Paulo/SP, Brazil.

elianabg@hcnet.usp.br

Received for publication on August 24, 2007.

Accepted for publication on September 27, 2007. quent antigenic variations. The smaller variations, known as "antigenic drifts", occur approximately each year and are responsible for the epidemics. The larger variations, "antigenic shifts", are less frequent and cause the great pandemics. Between 1977 and 1988, there were seven epidemics in the USA, with more than 10,000 deaths, with around $80 \%$ in individuals 65 years of age or older. ${ }^{3}$ The deaths, which can occur due to the action of the virus itself or are related to a secondary bacterial infection, most commonly occur in the elderly and individuals with chronic cardiopulmonary diseases.

The largest impact of influenza epidemics can be observed in terms of morbidity, especially in adults with respiratory disease, as infection causes higher hospitalization rates, increased numbers of ambulatory appointments, and absenteeism in work and school.

The use of vaccines of inactivated viruses since the mid- 
dle of the 1940's constitutes an important public health measure in order to reduce the occurrence of influenza and minimize its consequences.

Since 1948, the World Health Organization (WHO), through the Influenza Prevention Program, has coordinated a net of laboratories responsible for the surveillance and identification of the virus isolated in different continents. To date, the composition of the vaccine against influenza has been preconized and recommended for annual use during the autumn months in the north hemisphere.

Since 2000, the Ministry of Health in Brazil has implemented the System of Surveillance of Influenza. The system is designed to: perform viral monitoring, evaluate the impact of vaccination, and follow the tendencies of morbidity and mortality through a sentinel surveillance strategy involving health unities and diagnostic laboratories. $^{4}$

Many studies, mainly conducted in the Northern hemisphere, have evaluated the efficiency of the influenza vaccine, and have shown that the effectiveness of the vaccine in the reduction of severe disease, hospitalization, pneumonia, and death is superior (47 to $95 \%)^{5,6,7,8}$ to the reduction of the influenza occurrence (around 30\%). ${ }^{9}$ Although the effectiveness of influenza vaccination has not been extensively studied in Brazil, some Brazilian regional studies show that it is effective. ${ }^{10}$ Gross and collaborators, in a meta analysis evaluating 20 studies, verified that the influenza vaccine had the capacity of preventing hospitalization due to pneumonia in 32 to $45 \%$ of the elderly, to prevent death due to pneumonia and influenza in 31 to $65 \%$ of the elderly, and prevent death due to any cause in 27 to $30 \%{ }^{11}$ A systematic review of the efficacy and effectiveness of the Influenza vaccine in the elderly concluded that well matched vaccines prevented hospital admission for influenza and pneumonia, mainly in long-term health facilities. ${ }^{12}$ In Brazil, the influenza vaccine has been freely distributed by the public health system since 1999. Its coverage in the elderly from 2000 to 2005 in the Southeast and Southern regions was almost always inferior to the coverage in Brazil. In 2006, the vaccine coverage in the state of São Paulo was $80.2 \%$, the lowest in the Southeast region, while the coverage reached $85.7 \%$ in Brazil. ${ }^{13}$

This fact is paradoxical since the state of São Paulo has an adequately installed public health system and presents elevated vaccination routine coverage in other age groups. One of the factors that can contribute to this situation is the fear of adverse events related to this vaccine. Empirically, it is observed that the lay population, particularly the elderly and health care workers (HCW), associate several morbid states with the influenza vaccine and express a lot of uncertainty about its role in preventing influenza, which can contribute to lower adherence to the vaccination campaigns.

Analysis of the literature shows that the local reactions to the inactivated virus influenza vaccine are the most frequent. Erythema, pain, and edema in the region of the injection can occur around 12 to 24 hours after the vaccination. They are more frequent in adults and occur in $10 \%$ to $64 \%$ of individuals who are vaccinated. The systemic reactions in adults include fever, myalgia, arthralgia, headache, malaise, and lack of respiratory symptoms. They can appear in the first seven days after the vaccination and vary from less than $1 \%$ to $18.9 \%$, with an average of $10 \%{ }^{1}$

Although some neurological syndromes have been temporarily associated with the inactivated virus in the influenza vaccine, only Guillain-Barré syndrome was clearly associated with the swine virus vaccine used in $1976 .{ }^{14}$ In most recent years, it has been speculated that the reports of Guillain-Barré syndrome after influenza vaccination were not coincidental. Additional studies are necessary in order to investigate the possible relation between this syndrome and the influenza vaccine. ${ }^{15}$

Immediate hypersensitive reactions, such as angioedema, allergic asthma, or anaphylactic reaction, can rarely occur after influenza vaccination. These manifestations probably result from hypersensitivity to some component of the immunogen. The vaccination is only contraindicated in people who have presented severe adverse allergic reactions in previous doses.

The Immunization Center of the Hospital das Clínicas, São Paulo University Medical School participates in the National Vaccination Campaign against influenza, which occurs in the first semester of each year and vaccinates all individuals according to indications from the Ministry of Health; the subjects in our study consisted of a subpopulation of this cohort. Our study subjects were individuals aged 60 years or older and the health care workers (HCW) that had contact with elderly and immuno-depressed patients. The objective of this study was to contribute to the clarification of the causes related to the low coverage verified in the State of São Paulo by evaluating the occurrence of adverse events related to the influenza vaccine in a sample of the vaccinated population of the 2002 Campaign, constituted of elderly and HCW from the Hospital das Clínicas, São Paulo University Medical School.

\section{OBJECTIVE}

To investigate the occurrence of early adverse events (AE) after influenza vaccination. 


\section{CASUISTIC AND METHODS}

For the calculation of the sample, the program Statcalc - EpiInfo version 6.0 was used. It was estimated that in the year 2002, 4,000 people would be vaccinated. The expected frequency of adverse events in adults is $10 \% .{ }^{16} \mathrm{~A}$ sample of 200 individuals was calculated (confidence interval [CI=95\%]).

The selected population consisted of individuals who were 60 years of age or older, and the HCW from the Hospital das Clínicas, São Paulo University Medical School, were vaccinated against influenza at the Immunization Center of the Hospital das Clínicas, São Paulo University Medical School from April 13 ${ }^{\text {th }}, 2002$.

Inclusion criteria included:

- Elderly population: being 60 years old or older, or

- Health Care Worker population: being an active worker of the Hospital das Clínicas, São Paulo University Medical School, belonging to any age group.

When the subjects were 60 years old or more and a $\mathrm{HCW}$, they were added to the HCW group.

\section{Casuistics selection:}

This study was approved by the Ethics Commission for the Analysis of Research Projects from the Hospital das Clínicas, São Paulo University Medical School under number 321/02.

The first 200 people who met the inclusion criteria at the Immunization Center were contacted by telephone seven days after the vaccination. All participants included in the study agreed to answer a standardized inquiry via telephone.

The statistical analysis was executed by the EpiInfo version 6.0 program. In order to evaluate the differences in proportions, the $\mathrm{P}$ values were calculated using $\chi 2$ tests. $\mathrm{P}$ $<0.05$ indicated statistical significance.

\section{RESULTS}

From the 200 selected subjects, two were excluded because they were 59 years old and one because he did not agree to participate in the interview. From the 197 included, 146 were elderly and 51 were HCWs. In Table 1 , the distribution of the vaccinated subjects by gender is presented. The average age was 71 years (range: 60 to 90 years) among the elderly and 38.8 years (18 to 68 years old) among the HCWs.

The prevalence of previous asthma and bronchitis was used to characterize the subjects of the study, and it was not used to indicate AE. There was no statistically signifi- cant difference in the previous occurrence of asthma and bronchitis between the elderly and HCW. On the other hand, previous influenza vaccination was more frequent in the elderly (Table 2).

Local AE were reported by $32.5 \%$ and systemic AE were reported by $26.4 \%$ of the vaccinated. Additionally, we compared the occurrence of AE between the elderly and HCWs. The local and systemic AE frequency was more elevated among the HCWs than the elderly $(P<0.01)$.

As shown in Table 3, local AE were significantly more frequent among the HCWs due to the symptom of pain ( $P$ $<0.0001$ ) (Table 3).

Among the investigated systemic AE, headache, myalgia, malaise, and coryza were significantly more frequent in the HCW than the elderly (Table 4). The other investigated events (fever, asthenia, cough, dyspnea, expectoration, arthralgia, bronco spasm, thoracic pain, and rash) occurred in similar frequencies in both groups.

Table 1 - Distribution of individuals vaccinated against influenza according to gender. Hospital das Clínicas, São Paulo University Medical School. 2002

\begin{tabular}{lcccccc}
\hline \multirow{2}{*}{ Patients } & \multicolumn{2}{c}{ Male } & \multicolumn{2}{c}{ Female } & \multicolumn{2}{c}{ Total } \\
& $\mathrm{n}$ & $\%$ & $\mathrm{n}$ & $\%$ & $\mathrm{n}$ & $\%$ \\
\hline Elderly & 61 & 41.8 & 85 & 58.2 & 146 & 74.1 \\
HCW & 3 & 5.9 & 48 & 94.1 & 51 & 25.9 \\
Total & 64 & 32.5 & 133 & 67.5 & 197 & 100 \\
\hline
\end{tabular}

HCW - Health Care Workers; n-number; \%-percentage

Table 2 - Distribution of individuals vaccinated against influenza according to previous asthma, bronchitis and influenza vaccination. Hospital das Clínicas São Paulo University Medical School. 2002

\begin{tabular}{lccccc}
\hline Variable & \multicolumn{2}{c}{ Elderly } & \multicolumn{2}{c}{ HCW } & \multirow{2}{*}{$P$} \\
& $\mathrm{n}$ & $\%$ & $\mathrm{n}$ & $\%$ & \\
\hline Asthma / bronchitis & 11 & 9.6 & 2 & 3.9 & 0.212 \\
Previous vaccination & 126 & 87.5 & 30 & 58.8 & $<0.001$ \\
\hline
\end{tabular}

HCW - Health Care Workers; n-number; \%-percentage

Table 3 - Occurrence of local adverse events after influenza vaccination. Hospital das Clínicas, São Paulo University Medical School. 2002

\begin{tabular}{lccccccc}
\hline \multirow{2}{*}{ Local AE } & \multicolumn{2}{c}{ Elderly } & \multicolumn{2}{c}{ HCW } & \multicolumn{2}{c}{ Total } & $P$ \\
& $\mathrm{n}$ & $\%$ & $\mathrm{n}$ & $\%$ & $\mathrm{n}$ & $\%$ & \\
\hline Total & 37 & 25.3 & 27 & 52.9 & 64 & 32.5 & 0.00029 \\
Pain & 32 & 21.9 & 26 & 51.0 & 58 & 28.8 & $<0.0001$ \\
Erythema & 11 & 7.5 & 4 & 7.8 & 15 & 7.1 & 0.9 \\
Edema & 13 & 8.9 & 6 & 11.8 & 19 & 9.1 & 0.6 \\
\hline
\end{tabular}

AE-adverse events; HCW - Health Care Workers; n-number; \%- percentage 
Table 4 - Occurrence of systemic adverse events after influenza vaccination. Hospital das Clínicas, São Paulo University Medical School. 2002

\begin{tabular}{|c|c|c|c|c|c|c|c|}
\hline \multirow[t]{2}{*}{ Systemic AE } & \multicolumn{2}{|c|}{ Elderly } & \multicolumn{2}{|c|}{ HCW } & \multicolumn{2}{|c|}{ Total } & \multirow[t]{2}{*}{$P$} \\
\hline & $\mathrm{N}$ & $\%$ & $\mathrm{~N}$ & $\%$ & $\mathrm{~N}$ & $\%$ & \\
\hline Total & 30 & 20.5 & 22 & 43.1 & 52 & $26.4 \%$ & $<0.01 *$ \\
\hline Myalgia & 12 & $8.2 \%$ & 10 & $19.6 \%$ & 22 & $11.2 \%$ & $0.026^{*}$ \\
\hline Asthenia & 12 & $8.2 \%$ & 8 & $13.7 \%$ & 20 & $9.6 \%$ & 0.129 \\
\hline Malaise & 9 & $6.2 \%$ & 9 & $17.6 \%$ & 18 & $9.1 \%$ & $0.014 *$ \\
\hline Cough & 11 & $7.5 \%$ & 6 & $11.8 \%$ & 17 & $8.6 \%$ & 0.354 \\
\hline Cephalalgia & 6 & $4.1 \%$ & 10 & $19.6 \%$ & 16 & $8.1 \%$ & $<0.01 *$ \\
\hline Coryza & 8 & $5.5 \%$ & 8 & $15.7 \%$ & 16 & $8.1 \%$ & $0.022 *$ \\
\hline Fever & 7 & $4.8 \%$ & 3 & $5.9 \%$ & 10 & $5.1 \%$ & 0.761 \\
\hline Dyspnea & 4 & $2.7 \%$ & 4 & $7.8 \%$ & 8 & $4.1 \%$ & 0.112 \\
\hline Expectoration & 6 & $4.1 \%$ & 2 & $3.9 \%$ & 8 & $4.1 \%$ & 0.953 \\
\hline Arthralgia & 6 & $4.1 \%$ & 0 & 0 & 6 & $3 \%$ & 0.141 \\
\hline Bronco spasm & 2 & $1.4 \%$ & 2 & $3.9 \%$ & 4 & $2 \%$ & 0.266 \\
\hline Thoracic Pain & 2 & $1.4 \%$ & 0 & 0 & 2 & $1 \%$ & 0.405 \\
\hline Asthma & 1 & $0.7 \%$ & 0 & 0 & 1 & $0.5 \%$ & 0.553 \\
\hline
\end{tabular}

$\mathrm{AE}$ - adverse events; HCW - Health Care Workers; n-number; \%- percentage

Table 5 - Occurrence of local and systemic adverse events after influenza vaccination according to concomitant administration of other vaccines. Hospital das Clínicas, São Paulo University Medical School. 2002

\begin{tabular}{|c|c|c|c|c|c|}
\hline \multirow{3}{*}{$\frac{\mathrm{AE}}{\text { Local }}$} & \multicolumn{4}{|c|}{ Concomitant vaccination } & \multirow[t]{2}{*}{$P$} \\
\hline & \multicolumn{2}{|c|}{ Yes } & \multicolumn{2}{|c|}{ No } & \\
\hline & $\mathrm{N}$ & $\%$ & $\mathrm{~N}$ & $\%$ & \\
\hline yes & 15 & 27 & 49 & 36 & 0.25 \\
\hline no & 40 & 73 & 88 & 64 & \\
\hline \multicolumn{6}{|c|}{ Systemic } \\
\hline yes & 16 & 29 & 35 & 26 & 0.62 \\
\hline no & 39 & 71 & 102 & 74 & \\
\hline
\end{tabular}

There was not a statistically significant difference in the occurrence of local and systemic AE among the individuals exclusively vaccinated against influenza and the ones that received other vaccines concomitantly (Table 5).

\section{DISCUSSION}

In this study, the occurrence of early AE after influenza vaccination was investigated. Although severe $\mathrm{AE}$ were not observed, the obtained frequencies were higher than our initial estimates, which were based on the existing literature $^{1}$. As expected, the HCWs were younger than the elderly. The higher frequency of women among HCWs can be attributed to the gender distribution observed among health area workers in general and, in particular, in the Hospital das Clínicas, São Paulo University Medical School, which shows a predominance of the female gender.

Previous influenza vaccination, respiratory disease, and concomitant administration of other vaccines were not associated with local or systemic AE occurrence.
Local $\mathrm{AE}$ were more frequent than systemic $\mathrm{AE}$ in both investigated groups. It is noteworthy that $\mathrm{AE}$, both local and systemic, were more frequent in HCWs than in the elderly.

In relation to local $\mathrm{AE}$, pain, of subjective evaluation, was the only $\mathrm{AE}$ that was significantly more frequent amongst HCWs. Differences in the occurrence of edema and erythema, of objective evaluation, were not identified.

Concerning systemic AE, subjective symptoms were observed among the HCWs more frequently than in the elderly, whereas fever, of objective evaluation, was similar in both groups. The findings of this study do not confirm the common belief that credits the influenza vaccine with uncomfortable and severe adverse events.

This vaccine is effective in the prevention of influenza in around $90 \%$ of healthy young adults and in 30 to $40 \%$ among the elderly when the vaccinal strain is similar to the circulating strain. Under these circumstances, the vaccine is effective in the prevention of complications and death due to influenza. This effectiveness is not always understood and assimilated, not only by the lay population, but also by HCW.

Answering concerns from the target population in relation to the safety and efficacy of the influenza vaccine depends on ample disclosure of information regarding these issues, preferably obtained from studies performed in Brazil. Our group has previously conducted investigations on the effectiveness of the influenza vaccine in the elderly ${ }^{10}$, demonstrating a reduction in the amount of flu-like episodes among the vaccinated.

Taking into consideration the privileged role that HCWs have in the disclosure of information regarding health, and their potential influence (which may be negative) concern- 
ing the revelation of the benefits of this vaccine, we emphasize the importance of continuous data collection, as well as the disclosure of correct health information and results obtained by HCWs in national and international research as an additional strategy to elevate the influenza vaccine coverage rates during the annual vaccination campaigns.

\section{STUDY LIMITATIONS}

This study was hindered by the fact that it was originally planned to detect the occurrence of $\mathrm{AE}$ related to the influenza vaccine in subjects who were presumably healthy.
During the analysis, we detected that $\mathrm{AE}$ in $\mathrm{HCWs}$ were more prevalent than in the elderly. The results were validated by a statistically significant difference between groups. The authors thus decided to present those results, considering the role of HCWs from prominent institutions in influencing people in the community. Future studies specifically designed for this matter can improve the understanding of this subject.

The authors recognize the limitations of the use of information obtained by telephone interviews. In this study, we were interested in identifying $\mathrm{AE}$ by the words of the patients, and the telephone interview was a practical, inexpensive way of obtaining this information.

\section{REFERENCES}

1. Kilbourne ED, Arden NH. Inactivated Influenza Vaccines. In:VACCINES - Stanley A. Plotkin, Walter A. Orenstein. $-3^{\text {rd }}$ ed. Page 531-551. W.B. Saunders Company. Philadelphia, Pennsylvania. 1999.

2. Smith W, Andrewes CH, Laidlaw PP. A virus obtained from influenza patients LANCET 2:66-68, 1993. Mentioned by: Kilbourne ED, Arden NH. Inactivated Influenza Vaccines. In: VACCINES - Stanley A. Plotkin, Walter A. Orenstein. $-3^{\text {rd }}$ ed. Page 531-551. W.B. Saunders Company. Philadelphia, Pennsylvania. 1999.

3. Brammer L, Fukuda K, Arden NH. Influenza Surveillance - United States, 1992 -93 and 1993-94. MMWR. 1997;46:1-12.

4. http://portal.saude.gov.br/saude/ in 10/24/2006.

5. Ahmed AE, Nicholson KG, Nguyen VAN, Tam JS. Reduction in mortality associated with influenza vaccine during 1989-90 epidemic Lancet. 1995;346:591-595.

6. Beyer WE, Bruijn IA, Palache AM, Westerndorp RG, Osterhaus AD. Protection against influenza after analysis of serologic and field studies. Arch. Intern. Med. 1999;159:182-188.
7. Keitel WA, Cate TR, Couch RB, Huggins LL, Hess KR. Efficacy of repeated annual immunization with inactivated influenza virus vaccines over a five year period. VACCINE. 1997;15:1114-1122.

8. Simonsen L, Fukuda K, Schonberger LB, Cox NJ. The impact of influenza epidemics on hospitalizations. J. Infect. Dis. 2000;181:831837.

9. Patriarca PA, Weber TA, Parke RA, Hall WN, Kendal AP, Bregman DJ et al. Efficacy of influenza vaccine in nursing homes:Reduction in illness and complications during an influenza $\mathrm{A}\left(\mathrm{H}_{3} \mathrm{~N}_{2}\right)$ epidemic JAMA. 1985;253:1136-1139.

10. Gutierrez EB, Li HY, Santos ACS, Lopes MH. Effectiveness of influenza vaccination in elderly out patients in São Paulo City, Brazil. Rev Inst Med Trop São Paulo. 2001;43:317-320.

11. Gross PA, Hermogenes AW, Sacks HS, Lau J, Levandowski RA. The efficacy of influenza vaccine in elderly persons. A meta - analysis and review of the literature. Annals of Internal Medicine. 1995;123:518527. 
12. Jefferson T, Rivetti D, Rivetti A, Rudin M, Di Pietrantonj C, Demicheli V. Efficacy and effectiveness of influenza vaccines in elderly people: a systematic review. 2005;366:1165-74.

13. http:// pni.data.sus.gov .br/consulta_gripe.asp in 10/9/2006.

14. Safranek TJ, Lawrence DN, Kurland LT, Culver DH, Wiederholtm WC, Hayner $\mathrm{N}$ et al. Reassessment of the association between Guillain-Barre Syndrome and receipt of swine influenza vaccine in 1976-1977. Results of a two-state study. Am J Epidemiol. 1991;133:940-51.
15. Haber P, Destefano F, Ângulo FJ, Iskander J, Shadomy SV, Weintrub E et al. Guillain-Barré syndrome following influenza vaccination. JAMA. 2004;292:2478-81.

16. CDC. Prevention and control of influenza. MMWR Recommendations and Reports. 1999;48:1-28. 